\title{
Cardiopulmonary Mortality and Temperature
}

\author{
Mieczysław Szyszkowicz \\ Population Studies Division, Health Canada, Canada \\ Email: mietek.szyszkowicz@hc-sc.gc.ca
}

\begin{abstract}
The purpose of this study was to examine the associations between cardiopulmonary (CP) mortality and temperature in Canada. The recently developed statistical methodology was used. Mortality data (non-accidental death causes: $\mathrm{CP}$ and non-CP) recorded in 20 locations in Canada were used for the period of January 1, 1984 to December 31, 2007. Daily counts of deaths, temperature, and ozone levels were organized in the time-series data. Multivariate meta-analysis for non-linear multi-parameter associations was applied to estimate relative risk related to temperature exposure. Attributable mortalities related to heat and cold, with reference to optimum ambient temperature, associated with temperature were estimated by the locations. The study used the software elaborated in the $\mathrm{R}$ statistical language. The used statistical routines were developed by other authors. Attributable risks for temperature for CP mortality were estimated as: $7.6 \%(5.0,9.6)$, $7.1 \%(4.6,9.3), 0.5 \%(-0.1,1.0)$ for global, cold and heat period, respectively.
\end{abstract}

Keywords: Air pollution, cardiac, death, environment, mortality, respiratory, temperature

\section{Introduction}

The presented analysis is based on recent advances in statistical methodology on complex associations between mortality and temperature. This work is a kind of review study - it uses existing software accompanying two published papers on the mortality and temperature [1-2]. As an alternative input data for this software, Canadian data on mortality and temperature in 20 locations are used.

Climate change may result in variation of temperature levels. We might expect a shift of temperature from its accepted "normal" values or occurrences of unusual temperature sequences and events. A new pattern in temperature values, often their extreme values, raises important concerns for public health. Epidemiological studies have reported that extreme hot and cold temperatures have associations with an increase in daily emergency department (ED) visits, hospitalization, morbidity and mortality.

In one such type of study, a non-parametric approach was proposed to cluster the days using 15 parameters [3]. There were considered five ambient air pollutants lagged by 0,1 and 2 days. Thus each day was represented by a vector composed of 15 (3 lags for each of 5 air pollutants) values. Cluster algorithm allowed us to segregate the days into 8 different groups. The distribution of temperature was very different on the generated clusters. The "hot" and "cold" clusters were identified with the separation of temperature. Various health conditions (measured as ED visits) on the considered clusters have shown strong separations by their frequencies. The cluster with the largest number of days was used as the reference one. It is assumed that it represents the most common and frequent environment conditions. These types of results indicate that temperature affects health outcomes [3].

\section{Materials and Methods}

\subsection{Health Data}

Mortality data were collected from 20 locations in Canada over the years 1984-2007 (24 years, i.e. for 8,766 days in each location). Mortality outcomes were represented by daily counts of deaths for nonexternal causes only [4]. Deaths with primary cause related to cardio-pulmonary (CP) conditions were considered in this study as main focus on epidemiological mortality data in relation to temperature. Mortality data were classified with an underlying cause of death encoded using the International 
Classification of Diseases, 9th revision (ICD-9, World Health Organization (WHO), 1977), for deaths before 2000 and using ICD-10 (WHO, 1992) for deaths registered from 2000 onward. Under ICD-9, cardio-pulmonary mortality was considered to be a combination of circulatory and respiratory conditions, with ICD codes 390-520. Under ICD-10, cardiopulmonary is considered to be all codes beginning with I or $\mathrm{J}$, as well as a small number of $\mathrm{G}, \mathrm{M}$ and $\mathrm{R}$ codes, on the basis of expert physician advice. These cases were sub-groups of larger class non-external causes (ICD-9: 0-799 and ICD-10: A00-R99).

\subsection{Environmental Data}

In this study, ambient temperature is the major predictor variable. Mean daily temperature (measured in degree Celsius) was used as the exposure indicator. The daily values of temperatures were calculated by averaging the hourly data and next were averaged across the Environment Canada central monitor stations. The temperature data were calculated and assigned by location (20 cities) and day (8,766 days). Thus for specific location and day, the temperature data were linked with daily mortality data for this location and day.

In addition, ground-level ambient ozone was used in the meta-regression model. For each considered location in the study, average and range of ambient ozone was calculated based on daily values for 20 years. Daily maximum 8-hour average ozone concentration was used to represent levels by days in the study.

\subsection{Statistics}

As was already mentioned, this study used a set of $\mathrm{R}$ routines developed and presented by other authors and accompanied to their published papers [1-2]. The analysis is done with $\mathrm{R}$ software (version 3.2.4) using the packages $d l n m$ and mvmeta [1-2]. The corresponding codes used in this analysis, were retrieved from the personal website [5] of the first author of the publications [1-2].

Whole main statistical process follows steps presented in the study on mortality risk attributable to high and low ambient temperature from 384 locations in 13 countries [1]. It is a two-stage statistical process. The first step of the analysis uses standard time-series quasi-Poisson regression. The statistical models are fitted separately in each location (here 20). As the results the location-specific estimations of the relative risks $(\mathrm{RR})$ and their corresponding variances were obtained. In general, exactly the same specification, as in [1], was used in the first-stage of the applied methodology. A natural cubic B-spline of time with 8 degrees of freedom per year was incorporated to adjust for seasonal and long-terms changes, and in addition, an indicator of day of the week was also added in the formula. The association with temperature was modelled using a distributed lag non-linear model. The detailed description of the used methodology and software is given in the relative publications [1-2,5].

The minimum mortality temperature (MMT) corresponds to a minimum mortality percentile (MMP) of temperature, between the 1st and 99th percentiles. This value was derived from the best linear unbiased prediction of the overall cumulative exposure. This value was estimated separately by location and was used as the reference temperature. The MMT was considered as the optimum temperature. The total attributable risk was calculated in respect of this value [1].

\section{$3 \quad$ Results}

The results are described and presented in the form of three tables and four figures. Table 1 summarizes the number of deaths from each location by the considered causes of death: CP, non-CP, and All. Here the All group are mortality cases identified as deaths of non-external causes $(\mathrm{All}=\mathrm{CP}+$ non-CP). The table also shows MMP and MMT by three presented causes and locations in the study.

Figure 1 shows temperature ranges in the 20 locations in Canada considered in the study. The values of average temperature for 6 consecutive days (lag 0 - 5) were used. Vertical lines identify internal (continues) and boundary (dashed) knots used by natural splines in the statistical models [2]. 
Table 1. The number of deaths by cause (CP, non-CP, All) and the corresponding reference temperature (percentile, value). Canada, 1984-2007.

\begin{tabular}{|c|c|c|c|c|c|c|c|c|c|}
\hline & \multicolumn{3}{|c|}{$\mathrm{CP}$} & \multicolumn{3}{|c|}{ non-CP } & \multicolumn{3}{|c|}{ All } \\
\hline Location & Death & MMP & MMT & Death & MMP & MMT & Death & MMP & MMT \\
\hline Calgary & 41944 & 99 & 20.9 & 43425 & 73 & 11.8 & 85369 & 99 & 20.9 \\
\hline Durham & 23060 & 91 & 20.7 & 25025 & 82 & 18.4 & 48085 & 90 & 20.4 \\
\hline Edmonton & 41158 & 91 & 17.3 & 42327 & 1 & -26.9 & 83485 & 64 & $10.0-$ \\
\hline Halifax & 23919 & 43 & 5.0 & 26788 & 99 & 22.2 & 50707 & 93 & 19.4 \\
\hline Halton & 17321 & 91 & 21.8 & 19578 & 83 & 19.6 & 36899 & 83 & 19.6 \\
\hline Hamilton & 40320 & 91 & 21.8 & 43152 & 76 & 17.7 & 83472 & 91 & 21.8 \\
\hline London & 23989 & 91 & 21.8 & 27515 & 79 & 18.5 & 51504 & 90 & 21.4 \\
\hline Niagara & 40208 & 91 & 22.3 & 37605 & 77 & 18.8 & 77813 & 85 & 20.7 \\
\hline Ottawa & 46435 & 91 & 21.5 & 51043 & 80 & 18.3 & 97478 & 83 & 19.1 \\
\hline Peel & 25435 & 91 & 21.7 & 33217 & 79 & 18.3 & 58652 & 81 & 18.8 \\
\hline Regina & 14664 & 99 & 24.5 & 14298 & 1 & -29.3 & 28962 & 1 & -29.3 \\
\hline Sarnia & 10819 & 91 & 22.3 & 9533 & 81 & 19.3 & 20352 & 90 & 21.9 \\
\hline Saskatoon & 14963 & 85 & 17.3 & 14914 & 1 & -29.6 & 29877 & 87 & 17.9 \\
\hline SS-Marie & 7389 & 91 & 19.1 & 7804 & 91 & 19.1 & 15193 & 93 & 19.8 \\
\hline Sudbury & 13876 & 91 & 19.8 & 12974 & 78 & 16.0 & 26850 & 92 & 20.2 \\
\hline Toronto & 165415 & 59 & 12.2 & 198829 & 1 & -13.8 & 364244 & 61 & 12.9 \\
\hline Waterloo & 26667 & 91 & 21.0 & 27506 & 82 & 18.5 & 54173 & 93 & 21.6 \\
\hline Windsor & 20811 & 90 & 23.2 & 19015 & 77 & 19.8 & 39826 & 78 & 20.1 \\
\hline Winnipeg & 56402 & 82 & 17.9 & 58722 & 79 & 16.9 & 115124 & 80 & 17.3 \\
\hline York & 19647 & 91 & 21.5 & 23385 & 79 & 18.2 & 43032 & 80 & 18.5 \\
\hline
\end{tabular}

Note. CP: cardiopulmonary, All: CP + non-CP, The reference temperatures: MMP - minimum mortality percentile of temperature (\%), MMT - minimum mortality value of temperature $\left({ }^{\circ} \mathrm{C}\right)$.

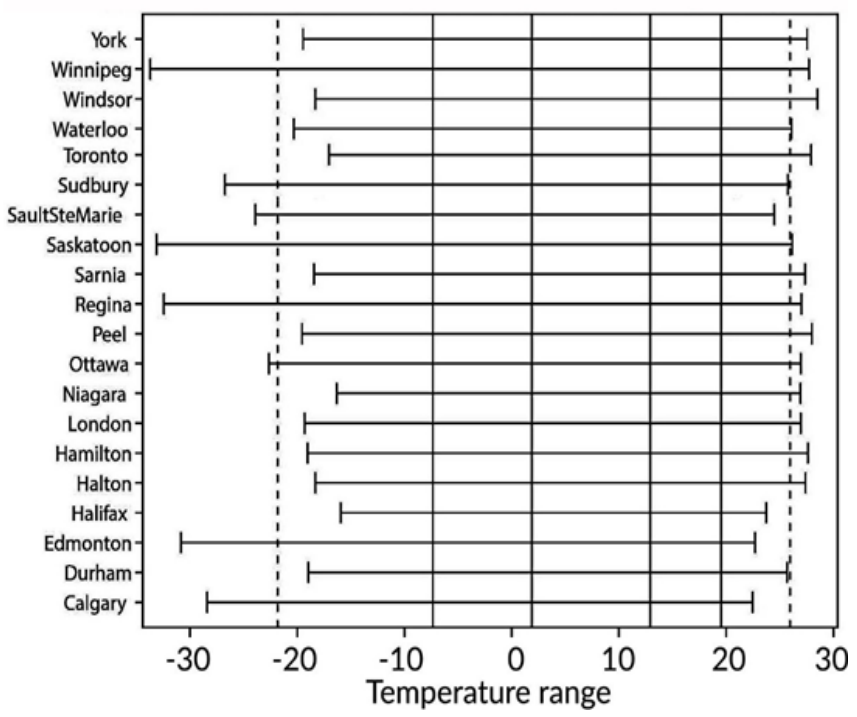

Figure 1. Range of temperature and the used knots. 



Figure 2. Relative risk by cause of death. Separation by two levels of ozone: 25 th and 75th percentiles, 27.6 and $34.5 \mathrm{ppb}$, respectively.

Figure 2 presents pooled and predicted exposure-response relationships in relative risk between relative temperature (percentiles) and three kinds of mortality in 20 locations in Canada, 1984-2007. The figure (left panel) shows the population average curve-response estimated in meta-analysis. The dots show locations of the used knots. The study specific curve-responses are also shown. The predicted curves from meta-analysis for the 25th and 75th percentiles of ambient ozone levels are shown (right panel) $[2]$.



Figure 3. Characteristics of the considered locations. Left Y-axis: latitude, ozone and MMT values, right Y-axis: attributable risk (in \%). CP as cause of death. 
Figure 3 provides additional information on the 20 locations. It shows the latitudes of the places used in the study, mean values of ambient ozone (daily level represented as average of O3 over 8 hours maximum), optimal temperature (MMT). In addition the figure shows (on the second (right) Y-axis) the estimated attributable risk for $\mathrm{CP}$ mortality. The corresponding numerical values are presented in Table 2 under CP in the column Total.

Table 2 shows the attributable risk for death by cause (CP, non-CP, and All) and period (overall, cold and warm).

Table 2. Attributable risk for death by cause and period. Canada, 1984-2007.

\begin{tabular}{c|c|c|c|c|c|c|c|c|c}
\hline & \multicolumn{3}{|c|}{ CP } & \multicolumn{3}{c|}{ Non-CP } & \multicolumn{3}{c}{ All } \\
\hline Location & Total & Cold & Heat & Total & Cold & Heat & Total & Cold & Heat \\
\hline Calgary & 2.4 & 2.5 & 0.0 & 2.6 & 2.1 & 0.6 & 1.7 & 3.5 & -0.1 \\
\hline Durham & 12.1 & 11.5 & 0.6 & 9.9 & 9.8 & 0.6 & 5.1 & 10.1 & 0.5 \\
\hline Edmonton & 6.0 & 5.8 & 0.3 & 8.8 & -0.1 & 9.2 & 0.8 & 1.2 & 0.3 \\
\hline Halifax & 4.1 & 1.7 & 2.4 & 18.1 & 19.5 & 0.0 & 4.3 & 8.8 & 0.0 \\
\hline Halton & 11.9 & 11.4 & 0.5 & 10.1 & 10.4 & 0.6 & 4.4 & 8.5 & 0.5 \\
\hline Hamilton & 7.6 & 7.2 & 0.4 & 5.7 & 4.7 & 1.3 & 2.1 & 3.7 & 0.6 \\
\hline London & 15.5 & 14.6 & 0.9 & 7.8 & 7.7 & 1.0 & 4.9 & 9.8 & 0.7 \\
\hline Niagara & 17.2 & 16.2 & 1.0 & 10.9 & 8.6 & 1.1 & 6.9 & 12.3 & 0.6 \\
\hline Ottawa & 12.1 & 11.6 & 0.5 & 7.8 & 7.5 & 0.8 & 5.7 & 11.1 & 0.6 \\
\hline Peel & 13.1 & 12.6 & 0.6 & 6.4 & 7.2 & 1.0 & 5.3 & 11.2 & 0.8 \\
\hline Regina & 2.8 & 2.8 & 0.0 & 5.6 & 0.0 & 5.6 & 2.3 & 0.0 & 4.7 \\
\hline Sarnia & 8.3 & 7.9 & 0.4 & 11.9 & 9.1 & 1.0 & 4.4 & 7.4 & 0.7 \\
\hline Saskatoon & 7.1 & 6.8 & 0.2 & 4.4 & 0.0 & 4.4 & 0.5 & 0.8 & 0.3 \\
\hline SS-Marie & 9.5 & 9.0 & 0.4 & 8.8 & 8.7 & 0.2 & 7.6 & 14.5 & 0.9 \\
\hline Sudbury & 12.2 & 11.7 & 0.5 & 5.0 & 4.0 & 0.6 & 4.4 & 7.9 & 0.4 \\
\hline Toronto & 2.4 & 2.0 & 0.4 & 7.0 & -0.1 & 8.6 & 1.6 & 2.3 & 1.2 \\
\hline Waterloo & 14.5 & 13.8 & 7 & 8.6 & 7.9 & 0.5 & 6.2 & 11.7 & 0.7 \\
\hline Windsor & 6.7 & 6.4 & 0.4 & 8.0 & 5.4 & 1.8 & 5.1 & 8.4 & 1.2 \\
\hline Winnipeg & 5.0 & 4.8 & 0.2 & 5.4 & 4.6 & 0.9 & 3.3 & 5.9 & 0.7 \\
\hline York & 13.4 & 12.9 & 0.5 & 7.1 & 7.2 & 1.1 & 5.4 & 10.7 & 0.9 \\
\hline Note. Abbrev & 10 & $1 n$ & 1.1 & 5 & & & & \\
\hline
\end{tabular}

Note. Abbreviations as in Table 1. The presented values are in the percentages.

Figure 3 provides additional information on the 20 locations. It shows the latitudes of the places used in the study, mean values of ambient ozone (average of O3 8hrmax), optimal temperature (MMT). In addition the figure shows (on the second (right) Y-axis) the estimated attributable risk for CP mortality. The corresponding numerical values are presented in Table 2 under CP in the column Total.

Figure 4 shows the relative risk curve-shapes obtained for the Edmonton city for two considered causes of deaths (CP and non-CP). 




Figure 4. Edmonton, Canada. Relative risk (with $95 \%$ empirical CI, shaded grey) for CP and non-CP. MMT (dotted line) and dashed lines indicate $2.5^{\text {th }}$ and $97.5^{\text {th }}$ percentiles. $\mathrm{RR}=$ relative risk.

Table 3 shows the results for the CP mortality obtained from the meta-regression approach wherein the used model, in addition to temperature and its range, was also added ambient ozone and range of the ozone.

Table 3. Adjusted for ozone in the meta-regression model. Attributable risk for CP mortality and the corresponding reference temperature. Canada, 1984-2007.

\begin{tabular}{c|c|c|c|c|c}
\hline Location & Total & Cold & Heat & MMP & MMT \\
\hline Calgary & 2.2 & 0.8 & 1.4 & 37 & 1.6 \\
\hline Durham & 15.4 & 14.9 & 0.5 & 90 & 20.4 \\
\hline Edmonton & 5.5 & 5.2 & 0.2 & 90 & 17.0 \\
\hline Halifax & 5.3 & 5.3 & -0.1 & 99 & 22.2 \\
\hline Halton & 14.4 & 13.7 & 0.7 & 92 & 22.1 \\
\hline Hamilton & 4.9 & 4.5 & 0.4 & 91 & 21.8 \\
\hline London & 14.7 & 13.7 & 1.0 & 91 & 21.8 \\
\hline Niagara & 16.6 & 15.6 & 1.0 & 91 & 22.3 \\
\hline Ottawa & 5.8 & 5.5 & 0.3 & 81 & 18.6 \\
\hline Peel & 14.4 & 14.0 & 0.4 & 89 & 21.0 \\
\hline Regina & 10.3 & 10.4 & -0.1 & 99 & 24.5 \\
\hline Sarnia & 13.5 & 12.6 & 0.9 & 91 & 22.3 \\
\hline Saskatoon & 1.9 & 1.7 & 0.2 & 78 & 15.2 \\
\hline SS-Marie & 13.3 & 12.6 & 0.7 & 91 & 19.1 \\
\hline Sudbury & 19.4 & 18.4 & 1.0 & 91 & 19.8 \\
\hline Toronto & 2.4 & 1.9 & 0.6 & 56 & 11.1 \\
\hline Waterloo & 16.8 & 16.0 & 0.8 & 91 & 21.0 \\
\hline Windsor & 8.3 & 8.0 & 0.3 & 90 & 23.2 \\
\hline Winnipeg & 5.0 & 4.8 & 0.2 & 81 & 17.6 \\
\hline York & 20.4 & 19.7 & 0.7 & 92 & 21.9 \\
\hline Abbreviat & & & & &
\end{tabular}

Note. Abbreviations and interpretations as in Tables 1 and 2.

There were $674,442 \mathrm{CP}$ deaths in total for the period $1984-2007$. Total average temperature was estimated as 6.6 (2.9-10.0) in Celsius degrees. The total estimated attributable fraction of CP deaths caused by cold and heat was $7.6 \%(95 \%$ eCI: $5.0,9.6) ; 7.1 \%(4.6,9.3)$ by cold, and by heat $0.5 \%(-0.1$, 
1.0). Here eCI is an empirical confidence interval [1]. Attributable risks for temperature with ozone were estimated as $7.8 \%(5.3,9.8), 7.3 \%(4.8,9.1), 0.5 \%(0.2,1.2)$, for total, cold and heat period, respectively.

\section{Discussion}

The results show that temperature is associated with substantial fraction of CP deaths. The attributable fraction is estimated as $7.6 \%$ of mortality within the study period. Higher value $(7.1 \%)$ is observed for days colder than the optimum temperature, than for days warmer than the optimum temperature $(0.5 \%)$.

The obtained results agree with the ones presented in the report of the study done in 384 locations in 13 countries [1].

This work also considers the impact of the ground-level ozone. Figure 2 shows large separation of RR by ozone levels for CP mortality. It is interesting that the ozone level decreased with latitude (Figure 3).

Figure 4 shows increased risks for cardiovascular and respiratory (CP) deaths with reference to the optimum temperature $\left(\mathrm{MMT}=17.3^{\circ} \mathrm{C}\right)$. For other causes of death (non-CP) the optimum temperature for Edmonton is lower negative $\left(\mathrm{MMT}=-26.9^{\circ} \mathrm{C}\right)$. Table 1 summarizes changes in MMT for $\mathrm{CP}$, non-CP and All by the considered locations. These results suggest the existence of various multiple biological pathways in relation to exposure to temperature for different health problems.

\section{References}

1. A. Gasparrini, Y. Guo, M. Hashizume, E. Lavigne, A. Zanobetti et al., "Mortality risk attributable to high and low ambient temperature: a multicountry observational study." The Lancet, vol. 386, no. 9991, pp. 369-375, 2015.

2. A. Gasparrini, B. Armstrong, M.G. Kenward, "Multivariate meta-analysis for non-linear and other multiparameter associations." Statistics in Medicine, vol. 31, no. 29, pp. 3821-39, 2012.

3. M. Szyszkowicz, E. Porada, E. Grafstein, "Ambient air conditions and emergency department visits in Vancouver." Advances in Environmental Research. Volume 39, Chapters Books. Nova Publishing, pp. 109-118. 2015.

4. M. Szyszkowicz, W.S. Burr, "The use of chained two-point clusters for the examination of associations of air pollution with health conditions." Int J Occup Med Environ Health, vol. 29, no. 4, pp. 613-22, 2016.

5. A. Gasparrini. Personal web page. Antonio Gasparrini: my research. http://www.ag-myresearch.com/r-code.html. 